\title{
Activation of acid-sensing ion channels by carbon dioxide regulates amygdala synaptic protein degradation in memory reconsolidation
}

\author{
Boren Lin ${ }^{1,2}$, Khaled Alganem³ ${ }^{3}$ Sinead M. O'Donovan³ ${ }^{3}$ Zhen Jin ${ }^{1}$, FarzanehSadat Naghavi ${ }^{3}$, Olivia A. Miller ${ }^{2}$, \\ Tyler C. Ortyl ${ }^{1}$, Ye Chun Ruan ${ }^{4}$, Robert E. McCullumsmith ${ }^{3,5}$ and Jianyang Du ${ }^{1,6^{*}}$ (D)
}

\begin{abstract}
Reconsolidation has been considered a process in which a consolidated memory is turned into a labile stage. Within the reconsolidation window, the labile memory can be either erased or strengthened. Manipulating acid-sensing ion channels (ASICs) in the amygdala via carbon dioxide $\left(\mathrm{CO}_{2}\right)$ inhalation enhances memory retrieval and its lability within the reconsolidation window. Moreover, pairing $\mathrm{CO}_{2}$ inhalation with retrieval bears the reactivation of the memory trace and enhances the synaptic exchange of the calcium-impermeable AMPA receptors to calcium-permeable AMPA receptors. Our patch-clamp data suggest that the exchange of the AMPA receptors depends on the ubiquitinproteasome system (UPS), via protein degradation. Ziram $(50 \mu \mathrm{M})$, a ubiquitination inhibitor, reduces the turnover of the AMPA receptors. $\mathrm{CO}_{2}$ inhalation with retrieval boosts the ubiquitination without altering the proteasome activity. Several calcium-dependent kinases potentially involved in the $\mathrm{CO}_{2}$-inhalation regulated memory liability were identified using the Kinome assay. These results suggest that the UPS plays a key role in regulating the turnover of AMPA receptors during $\mathrm{CO}_{2}$ inhalation.
\end{abstract}

Keywords: Carbon dioxide, Acid-sensing ion channels, Reconsolidation, Aversive conditioning, Memory retrieval, AMPA receptors, Ubiquitination, Proteasome, Protein degradation

\section{Introduction}

Being able to predict threats by recollecting fear stimuli is crucial for most animals such that one can behave adaptively to the dangerous environmental situation [1]. However, since fear memories developed due to traumatic events may trigger mental health conditions, attenuating such memories could be a solution to its resulting psychiatric disorders [2]. Pavlovian conditioning is a common method to develop a reflex response by training with repetitive actions. It was used to assess the ability of lab mice to learn and remember an association between

\footnotetext{
*Correspondence: jdu15@uthsc.edu

${ }^{1}$ Department of Anatomy and Neurobiology, The University of Tennessee Health Science Center, 38163 Memphis, TN, USA
}

Full list of author information is available at the end of the article a conditioned stimulus (CS, i.e. an auditory cue) and an aversive unconditioned stimulus (US, i.e. an electric foot shock), and how that memory can be modified [3]. A retrieval cue (CS alone) can recall and destabilize the previously coupled US-induced aversive memory, and at this labile state, the memory is subjected to diminish by the following extinction process, in which the animal receives consecutive auditory cues without coupling with foot shocks $[4,5]$.

Our earlier studies demonstrated that transient acidification by exposing animals to $\mathrm{CO}_{2}$ in conjunction with the retrieval cue significantly enhanced labilization of the target memory and more effectively weakened the aversive memory after memory extinction [6]. This regulatory effect was mediated by acid-sensing ion channels (ASICs), non-voltage gated $\mathrm{Na}^{+}$- and $\mathrm{Ca}^{2+}$-permeable original author(s) and the source, provide a link to the Creative Commons licence, and indicate if changes were made. The images or other third party material in this article are included in the article's Creative Commons licence, unless indicated otherwise in a credit line to the material. If material is not included in the article's Creative Commons licence and your intended use is not permitted by statutory regulation or exceeds the permitted use, you will need to obtain permission directly from the copyright holder. To view a copy of this licence, visit http://creativecommons.org/licenses/by/4.0/. The Creative Commons Public Domain Dedication waiver (http://creativeco mmons.org/publicdomain/zero/1.0/) applies to the data made available in this article, unless otherwise stated in a credit line to the data. 
channels that can be activated by extracellular acidosis [6]. During retrieval, increased rectification suggested that AMPA receptors (AMPARs) switched from $\mathrm{Ca}^{2+}$-impermeable (CI) to $\mathrm{Ca}^{2+}$-permeable (CP) to promote neuroplasticity, and $\mathrm{CO}_{2}$ inhalation during retrieval augmented the exchange of AMPA receptors and further energized $\mathrm{Ca}^{2+}$ influx [7]. Our data suggested that inhaling $\mathrm{CO}_{2}$ while the retrieval tone was presented caused more rectification than retrieval alone. In contrast, adding $\mathrm{CO}_{2}$ to retrieval in $\mathrm{ASIC} 1 \mathrm{a}^{-1-}$ mice failed to increase rectification [6].

However, the molecular signaling pathway that involves the $\mathrm{CO}_{2}$-induced lability of the retrieved memory is still not clear. Recent studies in the reconsolidation window suggested that the UPS, which controls the majority of protein turnover in mammals, is required for synaptic protein degradation, synaptic plasticity, and long-term memory [8]. Upon retrieval in the reconsolidation window, the consolidated memory has been considered destabilized and requires new protein synthesis to restabilize the labile memory $[4,9]$. Many intracellular $\mathrm{Ca}^{2+}$-dependent and -independent signaling pathways ( e.g. mTOR, MAPK and PKA) mediated protein translation involve in reconsolidation and inhibition of these pathways results in alternation in long-term memory within the original memory trace [10-13]. Also, $\mathrm{Ca}^{2+}$ and its binding proteins have been shown required for the activation of the UPS [14].

Combined the backgrounds with our previous studies, we hypothesized that $\mathrm{CO}_{2}$ inhalation in memory retrieval enhances UPS, particularly in targeting AMPAR subunit GluR2 to increase the exchange of $\mathrm{Ca}^{2+}$ impermeableAMPARs (CI-AMPARs) to $\mathrm{Ca}^{2+}$-permeable-AMPAR (CP-AMPARs). The outcome of this work might update our current understanding of the mechanism of memory reconsolidation.

\section{Materials and methods \\ Mice}

Both male and female 9-12 weeks old C57BL6 mice and $\mathrm{ASIC}_{1} \mathrm{a}^{-1-}$ mice were randomly selected for the experiment groups. The C57BL6 mice were ordered from the Jackson Laboratory and maintained in our animal facilities. The ASICla ${ }^{-/-}$mice were gifted from Drs. Michael Welsh and John Wemmie's laboratories at the University of Iowa and maintained in our animal facilities. Experimental mice were maintained on a standard 12-hour light-dark cycle and received standard chow and water ad libitum. Animal care and procedures met the National Institutes of Health standards. The University of Tennessee Health Science Center Laboratory Animal Care Unit (Protocol \#19-0112) and University of Toledo
Institutional Animal Care and Use Committee (Protocol \#108791) approved all procedures.

\section{Auditory aversive conditioning and retrieval}

All mice underwent habituation by handling for $15 \mathrm{~min}$ each day, 3 days before aversive conditioning. On day 1 , mice were habituated to the aversive conditioning chamber for $7 \mathrm{~min}$ (Med Associates Inc.). Then, the mice were presented with six pure tones $(80 \mathrm{~dB}, 2 \mathrm{kHz}, 20 \mathrm{~s}$ each) paired with 6 -foot shocks $(0.7 \mathrm{~mA}, 2 \mathrm{~s}, 100 \mathrm{~s}$ interval $)$ in context $\mathrm{A}$. After aversive conditioning, the mice were returned to their home cage. On day 2, $50 \mu \mathrm{M}$ Ziram was injected into the amygdala of the mice (see details in surgery and chemical infusion next section). The mice were then returned to their home cage until recovery. One hour later, the mice were placed into a new environment (context B) and inhaled either air or $10 \% \mathrm{CO}_{2}(10 \%$ $\mathrm{CO}_{2}+21 \%$ oxygen, balanced with nitrogen) for $7 \mathrm{~min}$. Five minutes after inhalation of air or $10 \% \mathrm{CO}_{2}$, a 20-second pure tone was used to retrieve the aversive memory. The mice were then returned to their home cages. Ten minutes later, the mice were euthanized, and brain slices were dissected for patch-clamp and western-blot experiments. To evaluate the outcomes of freezing behavior, the percentage of freezing time during the pure tone presentation was scored automatically using VideoFreeze software (Med Associates Inc.) [6].

\section{Surgery and chemical infusion}

For the Ziram injection procedure, mice were anesthetized using an isoflurane anesthetic vaporizer, secured to a Model 942 stereotaxic instrument (KOPF instruments). $1 \mu \mathrm{L}$ Ziram (50 $\mu \mathrm{M}$, diluted in saline) was injected bilaterally into the amygdala (relative to bregma: $-1.2 \mathrm{~mm}$ anterioposterior; $\pm 3.5 \mathrm{~mm}$ mediolateral; $-4.3 \mathrm{~mm}$ dorsoventral) using a $10 \mu \mathrm{l} \mathrm{Hamilton}$ microsyringe and a WPI micro-4 microsyringe pump [6, 15]. Mice were then recovered for $1 \mathrm{~h}$ followed by the retrieval procedure and then the brain slices were dissected for patchclamp recordings and western-blot experiments. For the Clasto-Lactacystin $\beta$-lactone ( $\beta$-Lac, Cayman Chemical, Ann Arbor, MI) injection, mice were anesthetized with isoflurane through an anesthetic vaporizer, secured to a Model 942 stereotaxic instrument (KOPF instruments) and a cannula made from a 25-gauge needle was inserted bilaterally into lateral amygdala (relative to bregma: -1.2 $\mathrm{mm}$ anterioposterior; $\pm 3.5 \mathrm{~mm}$ mediolateral; $-4.3 \mathrm{~mm}$ dorsoventral) $[6,15]$. Dental cement secured the cannula and bone anchor screw in place. Mice recovered for 4-5 days before any subsequent testing was carried out. A $1 \mu \mathrm{L}$ Hamilton syringe connected to a 30 -gauge injector was inserted $1 \mathrm{~mm}$ past the cannula tip to inject $0.3 \mu \mathrm{L} \beta$-Lac at $32 \mathrm{ng} / \mu \mathrm{l}$ (dissolved in DMSO in $1 \mu \mathrm{l}$ and 
further diluted in PBS) bilaterally [16, 17]. The injection sites were mapped post-mortem by sectioning the brain (10 $\mu \mathrm{m}$ coronal) and performing cresyl violet staining.

\section{Brain slice preparation and patch-clamp recording of amygdala neurons}

Ten minutes after the memory retrieval procedure, mice were euthanized with overdosed isoflurane, and the whole brains were dissected into pre-oxygenated $\left(5 \% \mathrm{CO}_{2}\right.$ and $\left.95 \% \mathrm{O}_{2}\right)$ ice-cold high sucrose dissection solution containing (in $\mathrm{mM}$ ): 205 sucrose, $5 \mathrm{KCl}$, $1.25 \mathrm{NaH}_{2} \mathrm{PO}_{4}, 5 \mathrm{MgSO}_{4}, 26 \mathrm{NaHCO}_{3}, 1 \mathrm{CaCl}_{2}$, and 25 glucose [6]. $300 \mu \mathrm{m}$ sections were dissected using a vibratome (Leica VT-1000 S) and incubated in ACSF containing (in mM): $115 \mathrm{NaCl}, 2.5 \mathrm{KCl}, 2 \mathrm{CaCl}_{2}, 1 \mathrm{MgCl}_{2}$, $1.25 \mathrm{NaH}_{2} \mathrm{PO}_{4}, 11$ glucose, $25 \mathrm{NaHCO}_{3}$ bubbled with $95 \% \mathrm{O}_{2} / 5 \% \mathrm{CO}_{2}, \mathrm{pH} 7.35$ at room temperature (20$22{ }^{\circ} \mathrm{C}$ ) at least $1 \mathrm{~h}$ before patch-clamp recordings. Slices were continuously perfused with $5 \% \mathrm{CO}_{2} / 95 \% \mathrm{O}_{2}$ ACSF $(\sim 3.0 \mathrm{ml} / \mathrm{min})$ in a recording chamber throughout the experiments [6].

Pyramidal neurons in the lateral amygdala were selected under an Olympus BX51WI upright microscope. The pipette solution for whole-cell patchclamp recordings contains (in $\mathrm{mM}$ ): $135 \mathrm{Cs}-\mathrm{SO}_{3} \mathrm{CH}_{3}$, $5 \mathrm{NaCl}, 10$ HEPES, 4 MgATP, $0.3 \mathrm{Na}_{3}$ GTP, 0.5 K-EGTA $(\mathrm{mOsm}=290$, adjusted to $\mathrm{pH} 7.25$ with $\mathrm{CsOH})$. The pipette resistance (measured in the bath solution) was 4-6 M . High-resistance (>1 G $\Omega$ ) seals were formed in voltage-clamp mode. Picrotoxin $(100 \mu \mathrm{M})$ was added to the ACSF throughout the recordings to avoid inhibitory responses. To record the AMPAR rectification and 1-naphthylacetyl spermine (NASPAM) sensitive currents, $100 \mu \mathrm{M}$ D-2-amino-5-phosphonovalerate (D-APV) was added to block the NMDAR-conducted excitatory postsynaptic currents (EPSCs). The EPSCs were measured ranging from -80 to $+60 \mathrm{mV}$ with a $20 \mathrm{mV}$ step. The ratio of the amplitude of EPSCs at -80 and $+60 \mathrm{mV}$ was measured to determine the rectification index. 50 $\mu \mathrm{M}$ NASPM was added to the bath solution to detect the NASPAM sensitive AMPAR-EPSCs, voltage was holding at $-80 \mathrm{mV}$. EPSCs were acquired at $10 \mathrm{kHz}$ using Multiclamp $700 \mathrm{~B}$ and pClamp 10.1. EPSCs were analyzed using Clampfit 10.1 [6].

\section{Immunoprecipitation (IP) and Western-blot analyses}

Tissue lysates were prepared by homogenization using pellet pestles in cold IP lysis buffer composed of $25 \mathrm{mM}$ Tris- $\mathrm{HCl} \mathrm{pH} \mathrm{7.4,} 150 \mathrm{mM} \mathrm{NaCl}, 1 \mathrm{mM}$ EDTA, $1 \%$ NP-40 and $5 \%$ glycerol with protease and phosphatase inhibitors (Thermo Scientific, Waltham, MA) followed by centrifugation at $12,000 \mathrm{rpm}$ for $15 \mathrm{~min}$ at $4{ }^{\circ} \mathrm{C}$ to remove debris. GluR2 in the whole cell lysate was concentrated by IP using monoclonal anti-GluR2 antibody and protein A/G agarose, both obtained from Thermo Scientific. To prepare synaptic protein extracts, tissue samples were homogenized in Syn-PER in synaptic protein extraction reagent (Thermo Scientific, Waltham, MA) on ice followed by serial centrifugations per manufacturer's instructions to isolate synaptosomes.

For Western-blot detection, samples were normalized for equal protein loading, boiled in sodium dodecyl sulfate (SDS) Laemmli sample buffer, resolved by SDS-polyacrylamide gel electrophoresis, transferred to polyvinylidene difluoride membranes and immunoblotted with antibodies specific for the proteins of interest. Polyclonal anti-phospho-Rpt6 antibody was obtained from Signalway, College Park, MD, and polyclonal antiCaMKII and anti-Actin antibody and mouse control IgG from Cell Signaling, Danvers, MA.

\section{0s proteasome activity assay}

Activities of the catalytic core of the proteasome complex 20s were measured by a 20s proteasome activity kit (MilliporeSigma, Burlington, MA) utilizing a fluorophore-labeled 20s substrate LLVY-AMC. Tissue lysates were prepared by homogenization using pellet pestles in cold T-PER tissue protein extraction reagent (Thermo Scientific, Waltham, MA) followed by centrifugation at $12,000 \mathrm{rpm}$ for $15 \mathrm{~min}$ at $4{ }^{\circ} \mathrm{C}$ to remove debris. The assay was performed according to the manufacturer's instructions in a 96-well fluorometer plate. Some samples collected from animals without behavior treatment were pre-incubated with a proteasome inhibitor Lactacystin for $15 \mathrm{~min}$ at room temperature before adding 20s substrate. After a $2 \mathrm{~h}$ incubation at $37^{\circ} \mathrm{C}$, fluorescence was measured at $380 / 460 \mathrm{~nm}$.

\section{Kinome assay}

All materials and software were obtained from PamGene, 's-Hertogenbosch, Netherlands. The kinase activity profiling platform, PamStation12, was used to measure serine/threonine kinome activity using the STK PamChip. Each array on the STK PamChip contains 141 serine/ threonine reporter peptides that are immobilized on a porous membrane. The assay was performed based on the PamStation12 standard protocols. Briefly, Pooled samples were diluted to $0.2 \mu \mathrm{g} / \mu \mathrm{L}$ and ran in duplicates on the kinome array, using 2 STK chips. Identical protein amounts were loaded for each condition. It was performed by blocking each well with $2 \%$ bovine serum albumin (BSA) before $2 \mu \mathrm{g}$ of protein of the samples, and $157 \mu \mathrm{M}$ adenosine triphosphate (ATP), and a primary antibody mixture as a part of the two-step reaction process specifically designed for STK PamChips. FITClabeled anti-phospho serine-threonine antibodies were 
added to each well. The homogenized samples alongside the assay mix were pumped through the wells through timed cycles to speed up the phosphorylation reaction between kinases in the samples and the reporter peptides on the chip. The degree of phosphorylation for each peptide in each well was measured in real-time using Evolve kinetic image capture software. The Evolve software captures images of FITC-labeled anti-phospho antibodies binding to each phosphorylated peptide substrates. Peptide spot intensities were captured across multiple exposure times $(10,20,50,100,200 \mathrm{~ms})$ during the post-wash phase. The BioNavigator software was used to convert the captured images to numerical values based on the intensity levels to be used for the kinome analysis.

The Kinome Random Sampling Analyzer (KRSA) software tool was used to process, analyze, and visualize the kinome data [18]. KRSA was used to calculate the linear regression slope (signal to exposure time) and scale the values by multiplying them by 100 . The logged transformed values were used as the final signal (i.e. peptide phosphorylation intensity) in comparative analyses. Quality control steps were used to remove peptides with either a very low signal $($ signal $<5$ ) or $R$-squared of less than 0.9 of the linear models. The signal ratio between pairs of group conditions was used to calculate $\log 2$ fold change (LFC) for each peptide. The LFC was calculated per chip and then averaged across the chips. Peptides with an LFC of at least 0.2 were carried forward to the upstream kinase analysis. For the upstream kinase analysis, KRSA takes these lists of peptides and uses a random resampling approach to identify implicated kinases using multiple kinase-substrate databases [18].

\section{Statistical analysis}

Nonparametric unpaired Mann-Whitney test was used to compare the mean results of two groups. One-way ANOVA and Tukey's post-hoc multiple comparison tests were used for comparing results more than two groups. $\mathrm{p}<0.05$ was considered statistically significant and labeled as an asterisk in the figures. Graphpad Prism 8 software was used to analyze statistical data. Data were presented as means \pm SEM. Sample sizes (n) are indicated in the figure legends, and data are reported as biological replicates (data from different mice, different brain slices). Each group contained tissues pooled from 3 to 5 mice.

\section{Results}

Inhibition of ubiquitination prevents $\mathrm{CO}_{2}$ and retrieval-induced AMPAR-EPSC rectification

Protein degradation and synthesis are critically involved in the process of reconsolidation [4, 9]. Memory retrieval results in time-dependent endocytosis of AMPAR subunits GluR1 and GluR2, which is observed within $6 \mathrm{~h}$ after retrieval $[7,19,20]$. Our previous data suggested that $\mathrm{CO}_{2}$ inhalation in memory retrieval enhances the lability of the memory and boosts the efficiency of the memory erasure through enhancing the exchange from CI-AMPA receptors to CP-AMPARs [6]. However, little is known about the molecular mechanism involved in the $\mathrm{CO}_{2}$ inhalation induced AMPARs turnover. Recent evidence suggests that the increased UPS activity regulates protein degradation and is critical for regulating memory reconsolidation $[16,21]$. To further test if the UPS involves in the $\mathrm{CO}_{2}$ inhalation-induced protein turnover, we measured the rectification of AMPARs (the signature of the CP-AMPARs) after $\mathrm{CO}_{2}$ inhalation and retrieval with Ziram, a ubiquitin E1 ligase inhibitor [22]. Twentyfour hours after aversive conditioning, Ziram $(50 \mu \mathrm{M})$ or saline was injected into the amygdala bilaterally $1 \mathrm{~h}$ before the memory retrieval (Fig. 1a). The data of aversive conditioning and retrieval were shown in Fig. 1b, c. In the saline injection group, we found that $10 \% \mathrm{CO}_{2}$ inhalation paired with retrieval induces a strong current rectification of AMPARs, indicating a sufficient number of CP-AMPARs was produced [6]. Whereas, the rectification was reduced in the Ziram injection group (Fig. 1d). This data indicated that the production of CP-AMPARs is ubiquitination dependent. In addition, we found that Ziram inhibited less AMPAR-EPSC rectification in the $\mathrm{ASICla}^{-1-}$ neurons than that in the WT group, suggesting that the $\mathrm{CO}_{2}$-induced AMPAR turnover is ASIC1a dependent (Fig. 1e). To further confirm the above result, we applied a specific CP-AMPARs inhibitor, 1-naphthylacetyl spermine (NASPM), to test the CP-AMPAR EPSCs after memory retrieval and $\mathrm{CO}_{2}$ inhalation. Compared to the saline injection group, $50 \mu \mathrm{M}$ ziram decreased NASPM-sensitive EPSCs (Fig. 1f, g). This data supports the conclusion that $\mathrm{CO}_{2}$ inhalation and retrieval-induced CP-AMPARs are ubiquitination dependent.

\section{$\mathrm{CO}_{2}$ inhalation in aversive memory retrieval potentiates ubiquitination}

Switching CI-AMPARs to more excitatory CP-AMPARs via ubiquitination, endocytosis and proteasomal degradation of AMPAR subunit GluR2 at glutamatergic synapses is essential for neuroplasticity $[7,20]$. By introducing a CI-AMPAR endocytosis inhibitor injected into the mouse lateral amygdala before memory retrieval, we have demonstrated that the effect of $\mathrm{CO}_{2}$ on conditioned freezing in the spontaneous recovery and renewal memory tests was attenuated. This data implicated that the endocytosis of AMPARs driving the exchange of its $\mathrm{Ca}^{2+}$-impermeable to preamble form may be enhanced by $\mathrm{CO}_{2}$-induced acidification and ASICs [6]. Since the presence of subunit GluR2 determines AMPAR's $\mathrm{Ca}^{2+}$ 


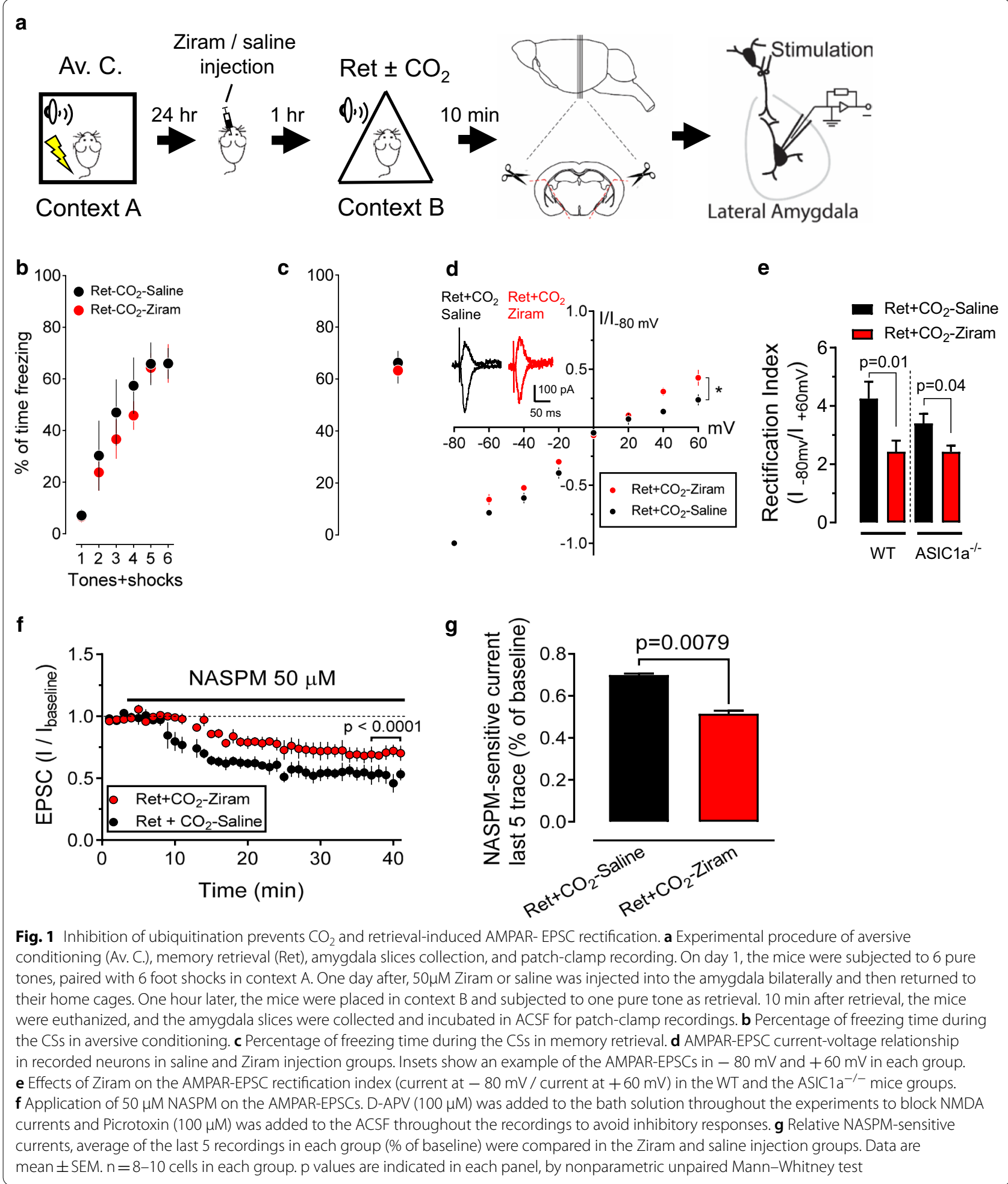

permeability, relevant molecular events on GluR2 were investigated in this study. Amygdala from mice receiving aversive conditioning, retrieval, and/or $10 \% \mathrm{CO}_{2}$ inhalation or sham controls were harvested, whole-cell and synaptosome lysates were prepared and Western blot analysis was conducted (Fig. 2a). The level of GluR2 
in the synaptosome suggested a decrease of this AMPAR subunit in response to $\mathrm{CO}_{2}$ inhalation (Fig. 2b). The ubiquitinated GluR2 was found more robust in mice exposed to $\mathrm{CO}_{2}$ during retrieval when a proteasome inhibitor was used to block the protein degradation (Fig. 2c).

Surprisingly, $\mathrm{CO}_{2}$ inhalation did not affect proteasome activity measured by 20 s core particle activity assays and Western blot analysis for phosphorylation of the 19s regulatory particle Rpt6 (Fig. 3). Although recent studies suggested that the proteasome activity was increased following aversive memory acquisition $[16,21]$, our data did not support that the increase of proteasome activity is required for either memory retrieval or $\mathrm{CO}_{2}$ inhalation. These results indicated that when the retrieval-induced lability of an aversive memory was enhanced by $\mathrm{CO}_{2}$ inhalation, ubiquitinmediated protein degradation on AMPAR subunit GluR2 was increased without changing the proteasome activity, leading to an increase of $\mathrm{Ca}^{2+}$ permeability of AMPARs.

a

Av. C.

Ret $\pm \mathrm{CO}_{2}$

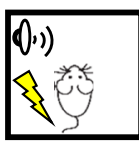

Context A
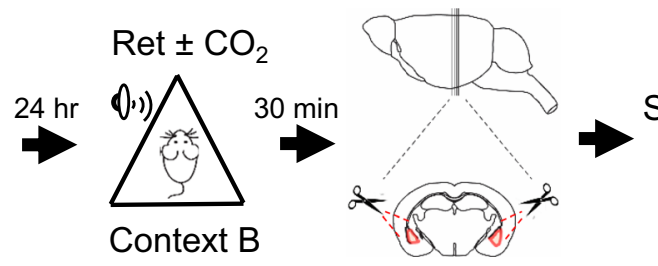

\section{Synaptosome Western \\ lysate $\rightarrow$ blot}

b

\section{Synaptosome Whole cell lysate}

$\begin{array}{llllll}\text { Av. C. } & + & + & + & + & \\ \text { Ret } & + & + & + & + & \mathrm{KDa} \\ \mathrm{CO}_{2} & - & + & - & + & \mathrm{KDa}\end{array}$

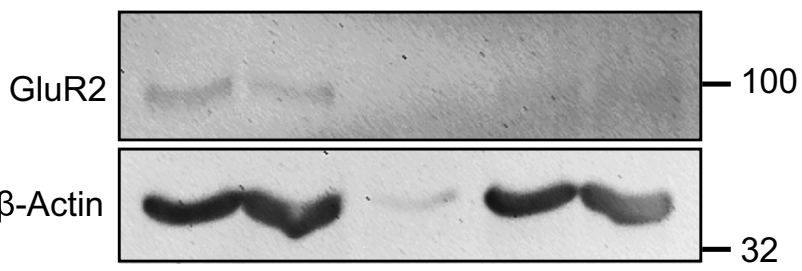

C

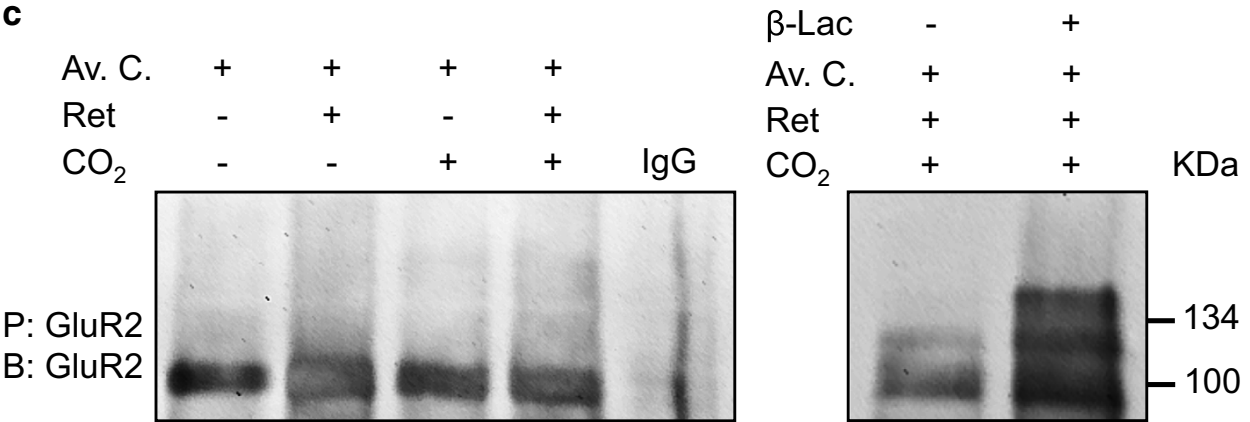

Fig. $2 \mathrm{CO}_{2}$ inhalation during retrieval promotes GluR2 ubiquitination and degradation. a Schematic of experimental procedure of aversive conditioning (Av. C.), memory retrieval (Ret) and $\mathrm{CO}_{2}$ inhalation, as described previously. Thirty minutes after, brain slices were prepared using a brain matrix, and amygdala isolated by a biopsy punch. Synaptosomal proteins were collected by using Syn-PER Reagent. Whole tissue lysates or synaptosomal proteins were subjected to SDS-PAGE and analyzed by Western blot. b Synaptosomal proteins collected from animals receiving aversive conditioning with or without Ret and with or without $\mathrm{CO}_{2}$ are indicated. GluR2 or actin was detected by using anti-GluR2 or anti-actin antibody respectively. c GluR2 in whole tissue lysates collected from animals subjected to aversive conditioning and Ret/ $\mathrm{CO}_{2}$ was first concentrated by IP using protein A/G agarose in conjunction with monoclonal anti-GluR2 antibody. Ubiquitinated GluR2 was assessed by Western blot analysis. Some animals were given $\beta$-Lac after behavior treatment. The negative control was prepared using nonspecific mouse lgG. Data are representative of three independent experiments 
a

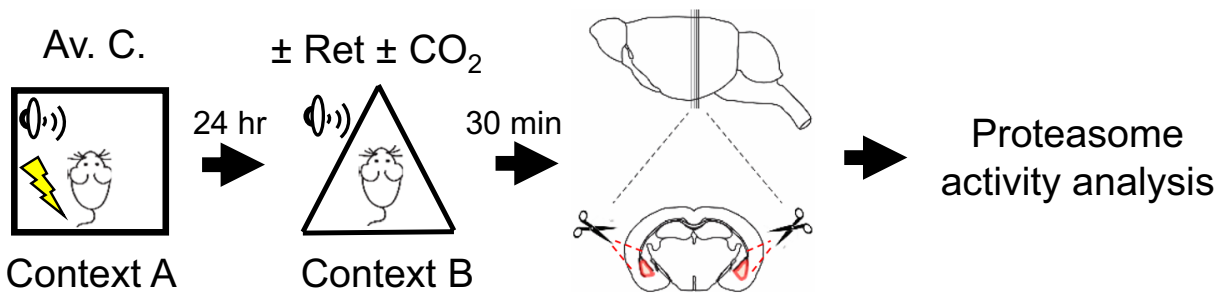

b

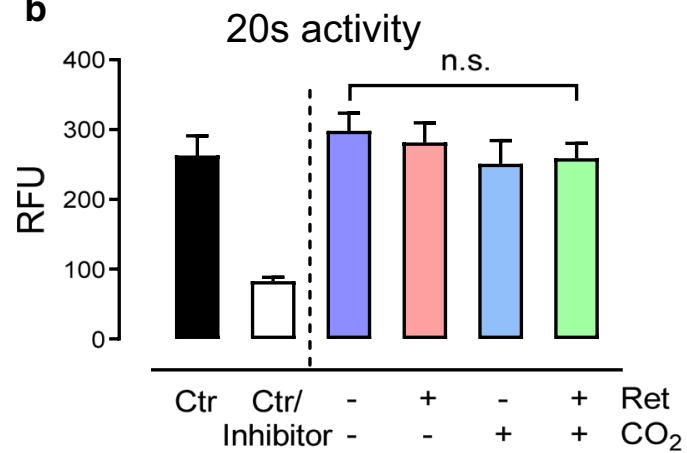

C

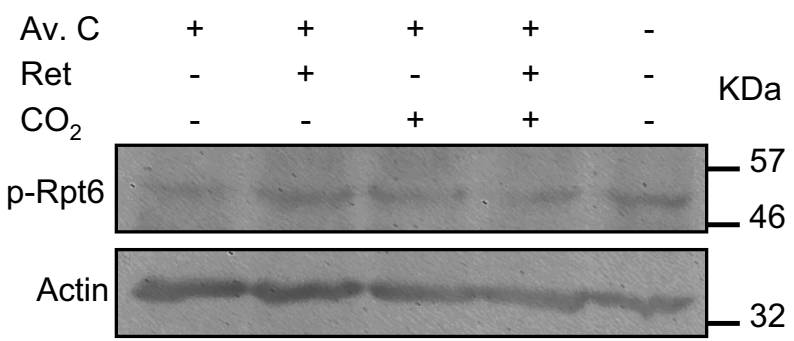

Fig. $3 \mathrm{CO}_{2}$ inhalation does not directly affect proteasome activity. a Schematic of experimental procedure of aversive conditioning (Av. C.), memory retrieval (Ret) and $\mathrm{CO}_{2}$ inhalation, as described previously. Thirty minutes later, brain slices were prepared using a brain matrix, and amygdala isolated by a biopsy punch. Whole tissue lysates were subjected to proteasome activity analysis. $\mathbf{b}$ Samples collected from animals receiving aversive conditioning with or without Ret and with or without $\mathrm{CO}_{2}$ are indicated. Proteasome activity in cell lysates was measured by a fluorometric proteasome 20s assay kit. Samples prepared from animals without behavioral treatment served as controls, and a proteasome inhibitor was used to confirm the specificity. c Tissue samples were collected from animals subjected to aversive conditioning and Ret/ $\mathrm{CO}_{2}$. Rpt6 phosphorylation was assessed by SDS-PAGE and Western Blot analysis using anti-phospho-Rpt6 and anti-actin antibodies. Values are expressed in mean \pm SEM of three independent experiments. 'n.s.' indicates not statistically significant by one-way ANOVA with Tukey's post-hoc multiple comparisons

\section{Potential $\mathrm{Ca}^{2+}$-dependent protein kinases that might be involved in the $\mathrm{CO}_{2}$ inhalation and retrieval-induced protein degradation}

Although intracellular $\mathrm{Ca}^{2+}$ is important for the $\mathrm{CO}_{2}$ inhalation enhanced memory lability and exchange of AMPARs [6], surprisingly, the activation of CaMKII, one of the kinases known to mediate signal cascades in learning and memory [23], was not further augmented (Fig. 4). To clarify how $\mathrm{Ca}^{2+}$ involves in the signaling pathways, we explored other $\mathrm{Ca}^{2+}$-dependent proteins. Since protein kinases are essential signaling components involved in various neuronal functions, we then investigated the potential impact on kinase activities due to transient acidification, amygdala tissues from mice subjected to aversive conditioning followed by memory retrieval with or without $\mathrm{CO}_{2}$ inhalation were harvested, lysed, and subjected to kinome analysis by using an array representing 141 reporter peptides as substrates for a wide spectrum of serine/threonine protein kinases (Fig. 5a) $[18,24]$. Phosphorylation on each peptide was detected in real-time using fluorescently labeled antibodies at different exposure times. The fold change in fluorescence intensity of each peptide between two sample groups was computed. Peptides that were undetectable, exhibited a non-linear signal-time relationship or showed an LFC lower than 0.2 were excluded. From these phosphorylated peptides, their responsible upstream kinases were identified, and among them, kinases with observed hits greater than two standard deviations from the distribution means (Z-Score $>2$ ), derived from 2000 iterations of random sampling analysis, were recognized as more active (retrieval vs. retrieval plus $\mathrm{CO}_{2}$ ) (Fig. 5b). SGK, DMPK and AMPK are $\mathrm{Ca}^{+}$-regulated kinases and were found more active in amygdala samples obtained from animals receiving $\mathrm{CO}_{2}$ inhalation (Fig. $5 \mathrm{c}-$ e), further supporting the role of ASICs for memory lability, and suggesting possible mediators of this process.

\section{Discussion}

In summary, our data suggest that (1) the $\mathrm{CO}_{2}$ inhalation-enhanced AMPAR turnover in reconsolidation window is UPS dependent; (2) $\mathrm{CO}_{2}$ inhalation enhances ubiquitin-mediated GluR2 degradation through the UPS without affecting the proteasome activity; (3) Although CaMKII is not involved in the $\mathrm{CO}_{2}$ inhalation-enhanced protein degradation, several 


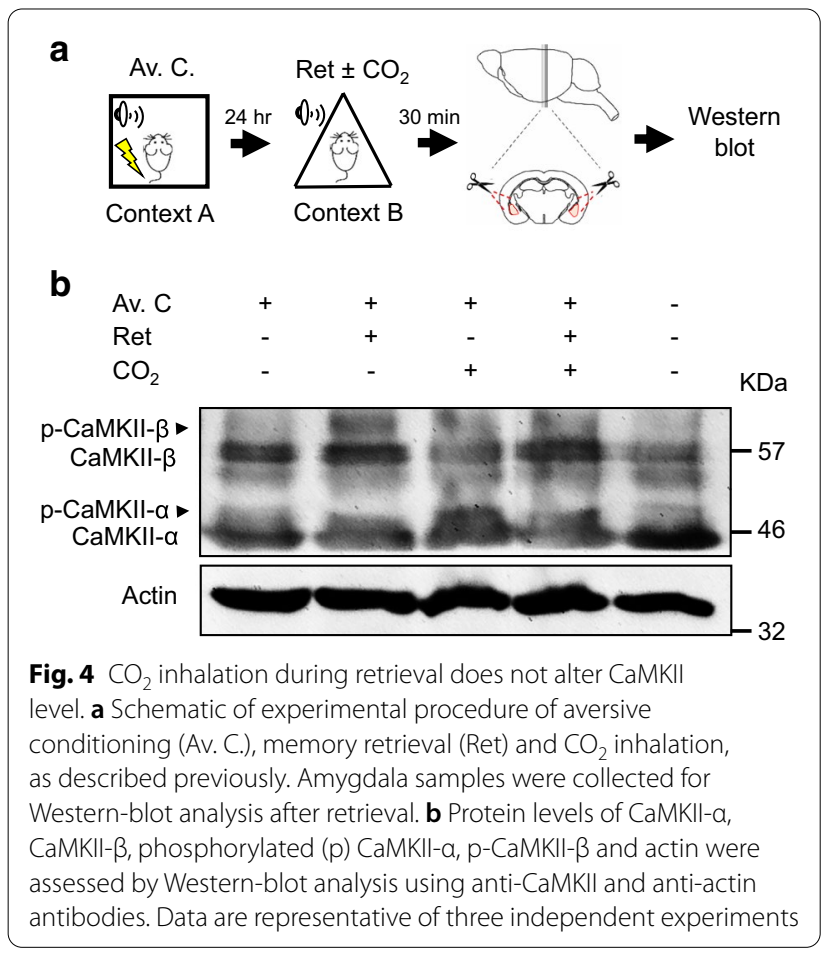

$\mathrm{Ca}^{2+}$-dependent kinases might be involved in the process. These results provide evidence to support the hypothesis that the application of $\mathrm{CO}_{2}$ inhalation, through activating ASICs, within the reconsolidation window enhances AMPAR turnover through promoting the intracellular $\mathrm{Ca}^{2+}$-dependent UPS (Fig. 6). Of course, we can not conclude that the effects of $\mathrm{CO}_{2}$ on protein degradation are entirely ASIC-dependent since the functions of $\mathrm{CO}_{2}$ inhalation in the brain are extensive. More works are necessary to identify the specific effects of $\mathrm{CO}_{2}$ on the reconsolidation process.

Numerous studies supported the hypothesis that a stabilized memory returns into a labile stage after memory retrieval called memory reconsolidation $[4$, $19,25]$. Protein degradation and new protein synthesis are involved in this process $[4,26]$. Recent evidence suggests that the UPS-dependent protein degradation is required for memory destabilization and updating in the amygdala and hippocampus $[16,27,28]$. In brief, ubiquitin is activated by the ubiquitin E1 ligase and then transferred to ubiquitin conjugases E2, and the complex interacts with a ubiquitin ligase E3, finally conjugated to lysine residues within the substrate, or the $\mathrm{N}$-terminal amino group. The complex then recognizes the targeted protein. The ubiquitinated targeted protein is recognized by the 26 s proteasome or lysosome leading to substrate degradation $[29,30]$. Thus, the activation of ubiquitylation and/or proteasome activity enhances the activity of the UPS. The retrieval of an aversive memory has been shown to activate the UPS through activating the proteasome activity in the amygdala [16]. In addition, CaMKII regulates the increases of both Rpt6-S120 phosphorylation and proteasome activity during memory reconsolidation [21].

In our previous studies, we have shown that $\mathrm{CO}_{2}$ inhalation during memory reconsolidation enhances the lability of the memory. The $\mathrm{CO}_{2}$ effects are through the activations of ASICs by which activating the intercellular $\mathrm{Ca}^{2+}$ signaling pathways, resulted in the turnover of AMPARs. The $\mathrm{CO}_{2}$ inhalation enhances memory retrieval within the reconsolidation window [6]. We thus asked if the $\mathrm{CO}_{2}$ inhalation and retrieval activate the same intracellular signaling pathways since both processes activated $\mathrm{Ca}^{2+}$-dependent signaling pathways. Interestingly, $\mathrm{CO}_{2}$ inhalation enhances the protein degradation without changing the proteasome activity through examining the proteasome activity and Rpt6S120 phosphorylation in the amygdala following memory retrieval. However, previous studies suggested that both the protein ubiquitination and proteasome activity are increased in the amygdala following memory acquisition and retrieval [16]. We then further test if $\mathrm{CO}_{2}$ affects the process of ubiquitination. Indeed, when inhibiting ubiquitin E1 ligase with $50 \mu \mathrm{M}$ Ziram, the exchange of the CI-AMPARs to CP-AMPARs has been decreased. This data suggest that $\mathrm{CO}_{2}$ inhalation enhances the reconsolidation through activating the ubiquitination process but not the proteasome activity. So, how does $\mathrm{CO}_{2}$ affect the

\footnotetext{
(See figure on next page.)

Fig. 5 Assessment of $\mathrm{CO}_{2}$ inhalation and retrieval-activated $\mathrm{Ca}^{2+}$-dependent protein kinases using Kinome assay. a Schematic of experimental procedure of aversive conditioning (Av. C.), memory retrieval (Ret) and $\mathrm{CO}_{2}$ inhalation, as described previously. Ten minutes later, brain slices were prepared using a brain matrix, and amygdala isolated by a biopsy punch. Whole tissues were subjected to kinome analysis. b A heatmap with hierarchical unsupervised clustering representing relative signal intensities of differentially phosphorylated peptides between memory retrieval and retrieval plus $\mathrm{CO}_{2}$ samples. Peptides were selected using 0.2 as the log2 fold change cutoff. c The linear model fit of the signal intensity as a function of the exposure time for three selected peptides. $\mathbf{d}$ The random sampling analysis output showing the expected distribution of mapped peptides to kinases based on a random selection of peptides (histogram) and the standard deviation (gray box represents two standard deviations from the mean) versus the observed number of peptides hits (red line). Red lines outside of the distribution indicate significant hits. e Log2 fold change values of peptides signals. Peptides that are mapped to AMPK, DMPK, and SGK show increased activities in the retrieval plus $\mathrm{CO}_{2}$ group
} 


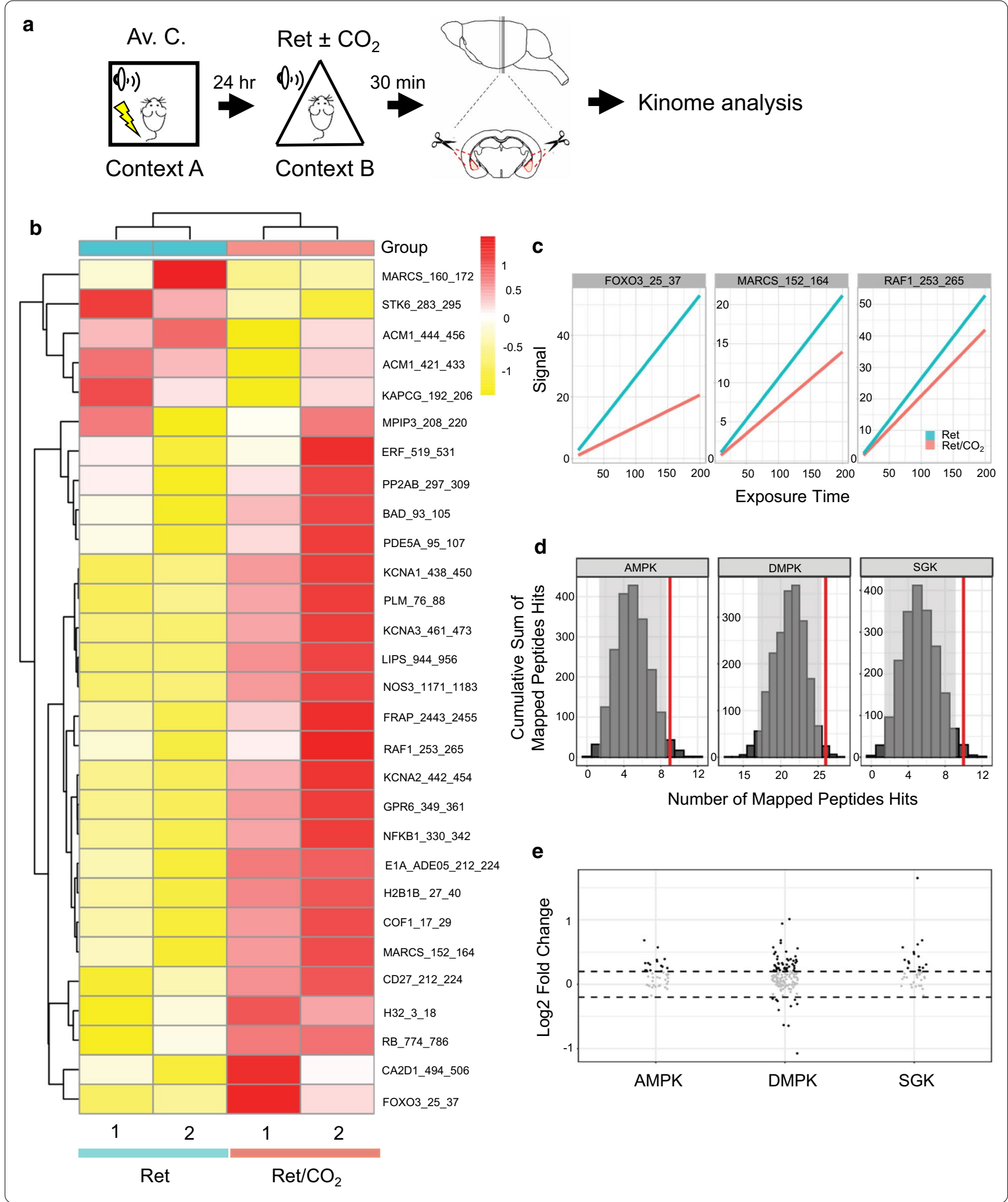

UPS efficiency in protein degradation without increasing the proteasome activity? Classically, ubiquitination is a signal that directs proteins to the proteasome, where the ubiquitin is recycled and the protein is degraded [31]. Also, ubiquitination recasts the surface of the targeted proteins and changes the protein's stability and activity 


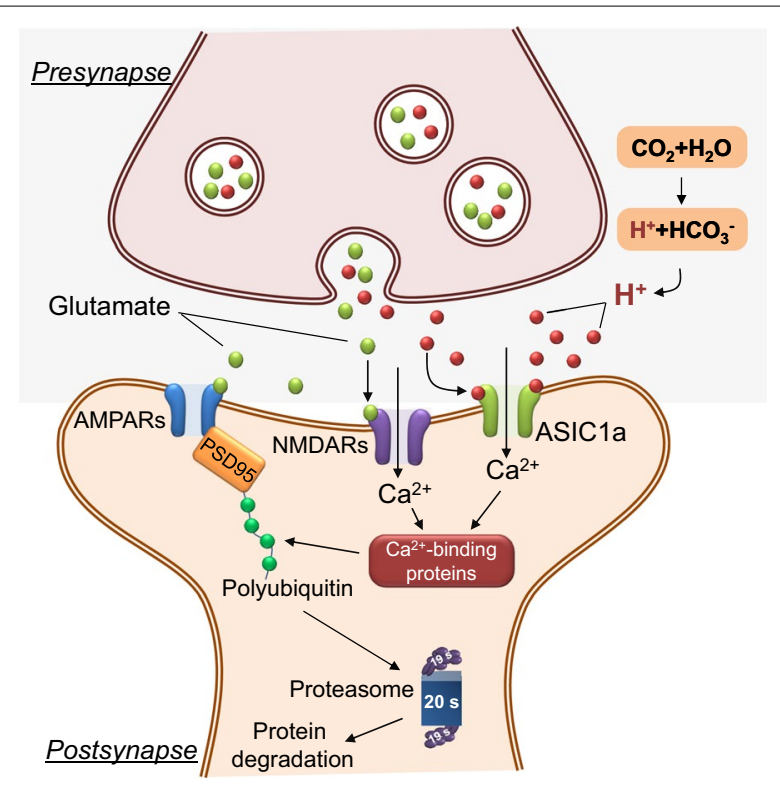

Fig. 6 A hypothesized model of how $\mathrm{CO}_{2}$ inhalation and ASICs regulate protein degradation after memory retrieval. $\mathrm{CO}_{2}$ inhalation decreases the brain $\mathrm{pH}$ during memory retrieval. The low $\mathrm{pH}$ activates ASICs on the postsynaptic membrane, results in the increase of intracellular $\mathrm{Ca}^{2+}$. Then $\mathrm{Ca}^{2+}$ can bind to its binding proteins and facilitate the ubiquitination process and eventually the polyubiquitinated GluR2 was degraded by the proteasome or other enzymes. The lack of GluR2 in the new synthesized AMPARs indicated the outcomes of the exchange of Cl-AMPARs to CP-AMPARs

[30], potentiates interactions with other proteins, and alters the subcellular localization [32]. Because ubiquitination might be particularly adapted to act as an efficient endocytic signal, the endocytosis of membrane AMPARs seems to be efficiently increased when enhancing the ubiquitin ligase E1.

Another interesting finding in this study is that the $\mathrm{CO}_{2}$ inhalation does not increase CaMKII phosphorylation after retrieval (Fig. 4). This is different from the mechanism of memory retrieval by which the CaMKII phosphorylation increases within the reconsolidation window [33]. Memory retrieval has been shown activated CaMKII phosphorylation to activate the AMPAR turnover. In addition, CaMKII regulates the UPS by activating the proteasome activity and the phosphorylation of the 19s regulatory subunit Rpt6, inducing protein degradation in the amygdala following memory retrieval [33]. Interestingly, evidence suggests that CaMKII only regulates the proteasome activities without directly affects the protein ubiquitination process [21]. We have previously shown that the effects of $\mathrm{CO}_{2}$ inhalation on memory retrieval are ASIC-dependent, which induces $\mathrm{Ca}^{2+}$ increase with the application of $\mathrm{CO}_{2}$. Of course, we cannot conclude whether the increase of intracellular $\mathrm{Ca}^{2+}$ was directly through ASIC1a or other $\mathrm{Ca}^{2+}$ channels. Because ASICs most likely form heteromeric sodium-selective channels and the calcium-permeable homomeric ASIC1a are limited in the brain. We used a powerful high throughput Kinome assay for kinase activity profiling in mice subjected to behavior treatment and found several $\mathrm{Ca}^{2+}$-dependent kinases with increased phosphorylation activity in response to $\mathrm{CO}_{2}$ inhalation in retrieval, suggesting potential signal molecules mediating acidification-induced signaling pathways in reconsolidation. Our studies in progress should further provide evidence elucidating how these kinases regulate UPS in such molecular events.

In all, our data demonstrated the cellular signaling pathway by which $\mathrm{CO}_{2}$ regulates the reconsolidation of aversive memories. Through activating ASICs, $\mathrm{CO}_{2}$ inhalation activates the ubiquitin mediated GluR2 degradation after memory retrieval without interfering with the proteasome activity in the amygdala neurons, different from the mechanism by which retrieval increases proteasome activity mediated by CaMKII (Fig. 6). Identifying the mechanism might have a substantial impact on our understanding of the mechanisms that underlie memory modification. This work is expected to lay the foundation for the development of novel therapies for mental illnesses such as post-traumatic stress disorder and anxiety.

\section{Abbreviations}

$\mathrm{CO}_{2}$ : Carbon dioxide; CS: Conditioned stimulus; US: Unconditioned stimulus; ASICs: Acid-sensing ion channels; AMPAR: a-Amino-3-hydroxy-5-methyl-4isoxazolepropionic acid receptor; CI-AMPAR: $\mathrm{Ca}^{2+}$ impermeabile-AMPAR; CP-AMPARs: $\mathrm{Ca}^{2+}$ permeable-AMPAR; GluR2: Ionotropic glutamate receptor 2; EPSC: Excitatory postsynaptic current; Av. C: Aversive conditioning; Ret: Retrieval; D-APV:: D-2-amino-5-phosphonovalerate; NASPM: 1-naphthylacetyl spermine; PCTX-1: Psalmotoxin-1; $\beta$-Lac: Clasto-Lactacystin $\beta$-lactone; CaMKII: $\mathrm{Ca}^{2+} /$ calmodulin-dependent protein kinase II; BSA: Bovine serum albumin; ATP: Adenosine triphosphate; KRSA: Kinome Random Sampling Analyzer; LFC: $\log 2$ fold change; IP: Immunoprecipitation.

\section{Acknowledgements}

We appreciate undergraduate students, Melissa Curtis, Nora Abdul-Aziz, Rida Naqvi, Caitlin Kilmurry, Becca Sturges, Jen Page, Chase Carr, Jordan Jones for their assistance.

\section{Author contributions}

B.L. and J.D. conceived the project. B.L., J.D. and Y.R. designed the experiments. B.L., Z.J., O.M., T.O. and J.D. performed the behavior and the patch-clamp experiments and data analysis. K.A., S.O., and R.M. performed the Kinome assay experiments and data analysis. Y.R. designed the signaling pathway image. B.L. and J.D. wrote the manuscript. All authors reviewed and edited the manuscript. All authors read and approved the final manuscript.

\section{Funding}

J.D. is supported by the National Institutes of Mental Health (R01 MH113986). R.M. is supported by the National Institutes of Mental Health (R01 MH107487). Y.R. is supported by the Early Career Scheme by Research Grants Council of Hong Kong (Y.C.R. No.24104517), Theme-based Research Scheme of Hong Kong (Y.C.R. No. T13-402/17 N). 


\section{Availability of data and materials}

All the data supporting the conclusions of this article are included within the manuscript. Original data files and any supporting materials are available upon request, from the corresponding authors.

\section{Declarations}

\section{Ethics approval and consent to participate}

All the experiments presented here were performed using mice. Animal care and procedures met the National Institutes of Health standards. The University of Tennessee Health Science Center Laboratory Animal Care Unit (Protocol \#19-0112) and University of Toledo Institutional Animal Care and Use Committee (Protocol \#108791) approved all procedures.

\section{Consent for publication}

Not applicable.

\section{Competing interests}

The authors declare no competing financial interests.

\section{Author details}

${ }^{1}$ Department of Anatomy and Neurobiology, The University of Tennessee Health Science Center, 38163 Memphis, TN, USA. ${ }^{2}$ Department of Biological Sciences, The University of Toledo, 43606 Toledo, OH, USA. ${ }^{3}$ Department of Neurosciences, The University of Toledo Medical Center, 43614 Toledo, $\mathrm{OH}$, USA. ${ }^{4}$ Department of Biomedical Engineering, Faculty of Engineering, The Hong Kong Polytechnic University, Hong Kong, People's Republic of China. ${ }^{5}$ Neurosciences Institute, ProMedica, OH 43614 Toledo, USA. ${ }^{6}$ Neuroscience Institute, The University of Tennessee Health Science Center, 38163 Memphis, TN, USA.

Received: 5 April 2021 Accepted: 26 April 2021

Published online: 07 May 2021

\section{References}

1. Ohman A, Mineka S. Fears, phobias, and preparedness: toward an evolved module of fear and fear learning. Psychol Rev. 2001;108(3):483-522.

2. Parsons RG, Ressler KJ. Implications of memory modulation for posttraumatic stress and fear disorders. Nat Neurosci. 2013;16(2):146-53.

3. Tovote P, Fadok JP, Luthi A. Neuronal circuits for fear and anxiety. Nat Rev Neurosci. 2015;16(6):317-31.

4. Nader K, Schafe GE, Le Doux JE. Fear memories require protein synthesis in the amygdala for reconsolidation after retrieval. Nature. 2000;406(6797):722-6.

5. Kim J, Song B, Hong I, Kim J, Lee J, Park S, et al. Reactivation of fear memory renders consolidated amygdala synapses labile. J Neurosci. 2010;30(28):9631-40.

6. Du J, Price MP, Taugher RJ, Grigsby D, Ash JJ, Stark AC, et al. Transient acidosis while retrieving a fear-related memory enhances its lability. Elife. 2017;6:e22564.

7. Hong I, Kim J, Kim J, Lee S, Ko HG, Nader K, et al. AMPA receptor exchange underlies transient memory destabilization on retrieval. Proc Natl Acad Sci U S A. 2013;110(20):8218-23.

8. Hershko A, Ciechanover A. The ubiquitin system. Annu Rev Biochem. 1998;67:425-79.

9. Tronson NC, Taylor JR. Molecular mechanisms of memory reconsolidation. Nat Rev Neurosci. 2007;8(4):262-75.

10. Duvarci S, Nader K. Characterization of fear memory reconsolidation. J Neurosci. 2004;24(42):9269-75.

11. Lubin FD, Sweatt JD. The IkappaB kinase regulates chromatin structure during reconsolidation of conditioned fear memories. Neuron. 2007;55(6):942-57.
12. Parsons RG, Gafford GM, Helmstetter FJ. Translational control via the mammalian target of rapamycin pathway is critical for the formation and stability of long-term fear memory in amygdala neurons. J Neurosci. 2006;26(50):12977-83.

13. Tronson NC, Wiseman SL, Olausson P, Taylor JR. Bidirectional behavioral plasticity of memory reconsolidation depends on amygdalar protein kinase A. Nat Neurosci. 2006;9(2):167-9.

14. VerPlank JJS, Goldberg AL. Regulating protein breakdown through proteasome phosphorylation. Biochem J. 2017;474(19):3355-71.

15. Koffman EE, Du J. Labeling aversive memory trace in mouse using a doxycycline-inducible expression system. Bio-protocol. 2017;7(20):e2578.

16. Jarome TJ, Werner CT, Kwapis JL, Helmstetter FJ. Activity dependent protein degradation is critical for the formation and stability of fear memory in the amygdala. PLoS One. 2011;6(9):e24349.

17. Ma N, Abel T, Hernandez PJ. Exchange protein activated by cAMP enhances long-term memory formation independent of protein kinase A. Learn Mem. 2009;16(6):367-70.

18. DePasquale EAK, Alganem K, Bentea E, Nawreen N, McGuire JL, Naji F, et al. KRSA: Network-based prediction of differential kinase activity from kinome array data. bioRxiv. 2020:2020.08.26.268581.

19. Monfils MH, Cowansage KK, Klann E, LeDoux JE. Extinction-reconsolidation boundaries: key to persistent attenuation of fear memories. Science. 2009;324(5929):951-5.

20. Clem RL, Huganir RL. Calcium-permeable AMPA receptor dynamics mediate fear memory erasure. Science. 2010;330(6007):1108-12.

21. Jarome TJ, Kwapis JL, Ruenzel WL, Helmstetter FJ. CaMKII, but not protein kinase A, regulates Rpt6 phosphorylation and proteasome activity during the formation of long-term memories. Front Behav Neurosci. 2013;7:115.

22. Monday HR, Bourdenx M, Jordan BA, Castillo PE. CB1-receptor-mediated inhibitory LTD triggers presynaptic remodeling via protein synthesis and ubiquitination. Elife. 2020;9.

23. Zalcman G, Federman N, Romano A. CaMKII isoforms in learning and memory: Iocalization and function. Front Mol Neurosci. 2018;11:445.

24. Bentea E, Depasquale EAK, O'Donovan SM, Sullivan CR, Simmons M, Meador-Woodruff $J \mathrm{H}$, et al. Kinase network dysregulation in a human induced pluripotent stem cell model of DISC1 schizophrenia. Mol Omics. 2019;15(3):173-88.

25. Schiller D, Monfils MH, Raio CM, Johnson DC, Ledoux JE, Phelps EA. Preventing the return of fear in humans using reconsolidation update mechanisms. Nature. 2010;463(7277):49-53.

26. Jarome TJ, Helmstetter FJ. Protein degradation and protein synthesis in long-term memory formation. Front Mol Neurosci. 2014;7:61.

27. Lee SH, Choi JH, Lee N, Lee HR, Kim JI, Yu NK, et al. Synaptic protein degradation underlies destabilization of retrieved fear memory. Science. 2008;319(5867):1253-6.

28. Lee $\mathrm{J}$. Memory reconsolidation mediates the strengthening of memories by additional learning. Nat Neurosci. 2008;11(11):1264-6.

29. Komander D, Rape M. The ubiquitin code. Annu Rev Biochem. 2012:81:203-29.

30. Pickart CM, Eddins MJ. Ubiquitin: structures, functions, mechanisms. Biochim Biophys Acta. 2004;1695(1-3):55-72.

31. Hershko A, Ciechanover A. The ubiquitin system for protein degradation. Annu Rev Biochem. 1992;61:761-807.

32. Mukhopadhyay D, Riezman H. Proteasome-independent functions of ubiquitin in endocytosis and signaling. Science. 2007;315(5809):201-5.

33. Jarome TJ, Ferrara NC, Kwapis JL, Helmstetter FJ. CaMKII regulates proteasome phosphorylation and activity and promotes memory destabilization following retrieval. Neurobiol Learn Mem. 2016;128:103-9.

\section{Publisher's note}

Springer Nature remains neutral with regard to jurisdictional claims in published maps and institutional affiliations. 